\title{
Misdiagnosis of Intestinal Tuberculosis as Crohn's Disease: A Case Report and Literature Review of Parametric Predictive Models
}

\section{Moussaoui Safa $^{1 *}$, M Zakhama ${ }^{1}$, M Moussaoui ${ }^{2}$, A Guediche ${ }^{1}$, I Jemni ${ }^{1}$, $N$ Ben Chaabène ${ }^{1}$ and L Safer ${ }^{1}$}

${ }^{1}$ Gastroenterology Department, CHU Monastir, Tunisia

${ }^{2}$ Radiology Department, CHU Monastir, Tunisia

*Corresponding Author: Moussaoui Safa, Gastroenterology Department, CHU Monastir, Tunisia.

\section{Received: September 01, 2021}

Published: September 20, 2021

(C) All rights are reserved by Moussaoui Safa., et al.

\begin{abstract}
In recent years, the incidence of Crohn's disease (CD) has been increasing worldwide, especially in areas where tuberculosis (TB) is prevalent. Due to similar manifestations, distinguishing between Crohn's disease (CD) and intestinal tuberculosis (ITB) is quite challenging.

Herein, we report the case of a 25-year-old man who presented with recurrent partial bowel obstruction, whose initial diagnostic work-up suggested stenosing Crohn disease 3 years ago. He had been previously treated with mesalazine and corticosteroids. After undergoing surgical treatment, histopathological findings revealed the diagnoses of intestinal tuberculosis instead.

We combined our case report with parametric predictive models reviewed in the literature to evaluate their diagnostic accuracy for our case. We found that using these models could lead to a better differentiation between these two entities.
\end{abstract}

Keywords: Crohn's Disease (CD); Tuberculosis (TB); Intestinal Tuberculosis (ITB)

\section{Background}

Crohn's disease (CD) and intestinal tuberculosis (ITB), although they have different etiology, pathogenesis, and therapeutic options, they can exhibit similar clinical, endoscopic, morphological and histological manifestations [1,2]. Therefore, differentiating these two entities is challenging for the clinician and can sometimes lead to misdiagnosis $[3,4]$. Here, we report a case whereby a male patient was diagnosed with ITB presenting with recurrent partial bowel obstruction, whose initial diagnostic work-up suggested CD. We compared our case with the results of the literature review using multiple multi-parametric predictive models.

\section{Patients and Methods}

We obtained the patient's signed permission for the publication during his follow-up consultation at our hospital. We performed a
Pub Med search using the terms "Crohn AND Intestinal tuberculosis," retrieving articles with parametric predictive models and scores to differentiate these two diseases. Furthermore, we combined this data with our case report to evaluate its diagnostic accuracy in our case.

\section{Presentation of Case}

A 25-year-old man was brought to the Emergency Room presenting with a four-month history of recurrent partial bowel obstruction, accentuated during the last 24 hours associated with right lower abdominal quadrant pain.

The patient had been diagnosed 3 years ago with stenosing Crohn's disease and had previously been treated with mesalazine and occasionally with corticosteroids during flare up. A surgery 
was proposed at that time due to short and symptomatic small intestine stenosis, but the patient refused and was lost to follow up. During the past 3 years, he hasn't received any treatment and reported recurrent subocclusif syndrome manifested by severe abdominal pain especially in the right lower abdominal quadrant accompanied by bloating during several minutes relieved by gas emission.

At the time of admission, the patient didn't complain of diarrhoea, fever or night sweats. The physical examination revealed tenderness at the right lower abdominal quadrant. The rest of examination was normal. Laboratory blood exams; Complete Blood Count, Basic metabolic panel, Comprehensive metabolic panel were in the normal range. QuantiFERON-TB test was positive.

An abdominal CT-scan was performed to exclude surgical emergency, displaying parietal circumferential thickening of the ileum and the cecum, extending over $76 \mathrm{~mm}$. The chest X-ray was normal. The patient had undergone a colonoscopy showing ileal stenosis and a pseudopolypoid ileocecal valve (Figure 1). The decision was to opt for surgical treatment and azathioprine intake to prevent post-operative relapse. The patient underwent a $21 \mathrm{~cm}$ terminal ileum resection.

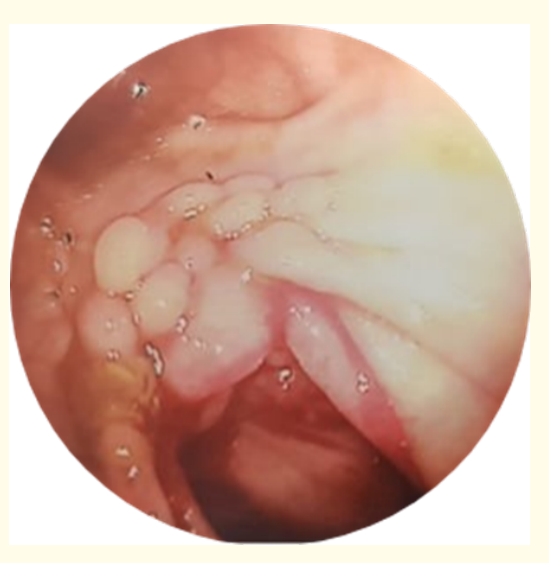

Figure 1: Colonoscopy showing ileal stenosis and pseudo-polypoid ileocecal valve.

The histopathological findings of the surgical specimen revealed the presence of numerous granulomas with necrotizing and calcified lymph node involvement. This led to reconsideration of TB as a differential diagnosis.

The key to diagnosis was performing a polymerase chain reaction (PCR) to identify Mycobacterium tuberculosis (MT) which came positive.

Ultimately, the diagnosis of ITB was confirmed and the patient began standard TB treatment regimen of rifampin, isoniazid, pyrazinamide, and ethambutol with significant response.

\section{Discussion}

The positive rate of specific methods for the diagnosis of TB and $\mathrm{CD}$ is low. In hesitant situations, the diagnosis cannot be definite. In recent studies, multivariable prediction models have been established to distinguish between CD and ITB [1].

We combined our case report with some parametric predictive models reviewed in the literature to evaluate their diagnostic accuracy in our case.

\section{Meta-analytic Bayesian model}

We assessed and used a Bayesian model [5] that produces estimates of the probability of ITB and CD that are calibrated to local prevalence. The score was available online [6].

The Bayesian model had promising results; sensitivity of $90.9 \%$, specificity of $92.6 \%$, and accuracy for diagnosis of ITB at $91.8 \%$.

This model is composed of different criteria: Prevalence of intestinal tuberculosis, Clinical manifestations, Endoscopic findings, Pathological findings, especially granuloma characteristics, CT scan findings and finally, Interferon gamma release assay.

The prevalence of TB in patients with small bowel ulceration is low in Tunisia, estimated at 0,05 which is similar to the US (we don't have baseline prevalence in Tunisia), Our patient had a probability of ITB estimated at $68,62 \%$.

\section{Model based upon endoscopic features}

A predictive model based upon endoscopic features was reported by Lee., et al. in 2006 [4]. They included eight features: transverse ulcers, fewer than 4 segment involvement, patulous ileocecal valve, and pseudopolyps (common in ITB), and longitu- 
dinal ulcers, aphthous ulcers, cobblestoning and anorectal lesions (common in CD).

Our patient had only two features consisting of involvement of less than 4 segments and the presence of pseudopolyps meaning a score of- 2 suggestive of ITB.

\section{Computerized tomography based score}

A predictive model published recently features computerized tomography results of ITB and CD [8]. This model is based on three characteristics (ileocecal area involvement, larger lymph nodes, and long-segment involvement) and had good specificity (90\%) and positive predictive value (80\%) in differentiating CD from ITB.

Our patient presented with ileocecal region involvement, one lymph node $\geq 1 \mathrm{~cm}$ and long-segment involvement (Figure 2), meaning a score of 1 , suggestive of ITB.

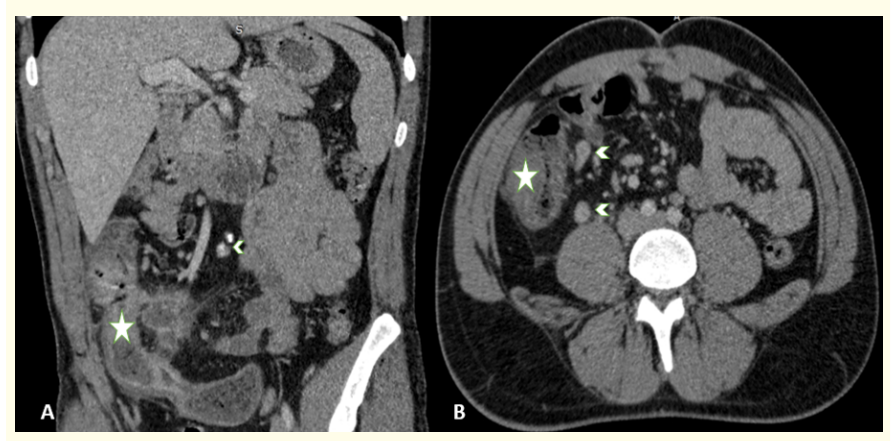

Figure 2: Coronal (A) and axial (B) CT images on portal venous phase demonstrate: ileocecal long segment involvement (star) and large lymph nodes (arrow head).

Korean model

Recently, Jung., et al. [8] proposed a model with good performance based on the results of a large retrospective cohort in seven centres in Korea, but the model has not been valid outside of Korea.

The variables selected for the predictive model were: age, gender, diarrhea, transverse ulcer, longitudinal ulcer, sigmoid colon involvement, and suspicion of pulmonary TB.

The optimal cut-off value for a predictive diagnosis was 0.350 in both models. Our patient had a score of 0,21 suggestive of CD.
In our case, most of the predictive models and scores mentioned in our review were suggestive of ITB except for the seven-marker risk score.

\section{Conclusion}

There have been till date many studies aiming to reach an ultimate equation capable of differentiating between CD/ITB. In the last few years, we have seen some new parameters on this aspect, including the latest Bayesian meta-analysis model, a model based upon endoscopic features, a CT based score, and the Korean model. Using these models could lead to a better differentiation between these two diseases.

\section{Bibliography}

1. Kedia S., et al. "Differentiating Crohn's disease from intestinal tuberculosis". World Journal of Gastroenterology 25 (2019): 418-432.

2. Lee YJ., et al. "Analysis of colonoscopic findings in the differential diagnosis between intestinal tuberculosis and Crohn's disease". Endoscopy 38.6 (2021): 592-597.

3. Wei JP., et al. "Misdiagnosis and mistherapy of Crohn's disease as intestinal tuberculosis case report and literature review". Med 95.1 (2016).

4. Akbar HO. “Intestinal tuberculosis and Crohn's disease: The dilemma of similarities and misdiagnosis". BMJ Case Reports (2009).

5. Limsrivilai J., et al. "Meta-Analytic Bayesian Model for Differentiating Intestinal Tuberculosis from Crohn's Disease". American Journal of Gastroenterology 112 (2017): 415-427.

6. Model to differentiate intestinal TB from Crohn's disease (2021).

7. Kedia S., et al. "Computerized tomography-based predictive model for differentiation of Crohn's disease from intestinal tuberculosis". Indian Journal of Gastroenterology 34.2 (2015): 135-143.

8. Jung Y., et al. "Predictive factors for differentiating between Crohn's disease and intestinal tuberculosis in Koreans". The American Journal of Gastroenterology 111.8 (2016): 11561164.

\section{Volume 4 Issue 10 October 2021} (C) All rights are reserved by Moussaoui Safa., et al. 